\title{
Macroscopic Quality Measurement of Plasma Treated Polystyrene through Computer Vision
}

\author{
F. Luthon *, F. Clément \# \\ University of Pau and Adour River, France \\ * Laboratoire d'Informatique, EA 3000 \\ \# Laboratoire d'Electronique des Gaz et Plasmas, EA 750 \\ IUT Château Neuf, Place Paul Bert \\ Avenue de l'Université BP1155 \\ 64100 Bayonne, France \\ 64013 Pau Cedex, France \\ Franck.Luthon@univ-pau.fr \\ Franck.Clement@univ-pau.fr
}

\begin{abstract}
Using a DC pulsed plasma for polymer surface treatment allows to obtain macroscopic modifications of the surface such as an important increase of the wettability i.e., reduction in hydrophobicity. In the same time, it yields microscopic variations of the surface structure that are mainly due to low depth chemical modifications even if only weak roughness changes appear. As a consequence this technique presents two major interests: an economical interest because of the low power consumption compared to other techniques like radio-frequency or microwave plasmas, and a non-invasive interest: very significant macroscopic treatment realized in soft conditions without degradation of the polymer. Applications are numerous: glue and painting production, health industry, aeronautics, wood industry, textile industry.

To characterize the benefits of this technique for polymer surface treatment, we present an image processing tool that yields macroscopic measures of wettability, through the estimation of drop contact angles. The process is automated, precise and low-cost.

The measurement of contact angle helps to infer microscopic properties from macroscopic features extracted via the unsupervised image processing technique. Results of macroscopic and microscopic studies on polystyrene surfaces allow to establish a macroscopic interpretation of the interaction between polymer and the DC pulsed plasma.
\end{abstract}

Keywords - Quality Measurement, Polystyrene, Thin Film, Surface Treatment, DC Pulsed Plasma, Wettability, Contact Angle, Edge detection.

\section{INTRODUCTION}

Improving the surface properties of polymers using a DC pulsed glow discharge in reactive gases is of great interest for industrial applications (e.g. glue, painting). This non polluting technique yields nice surface modifications such as an increase of wettability i.e., the ability to get wet [1]. The adhesion mechanisms are then improved even if the adhesion is not realized with water but more often with an epoxy adhesive. The very low energy cost of this treatment method is remarkable. Since the duration of the discharge corresponding to one DC pulse is one hundred times lower than the time period between two DC pulses, polymer samples are mainly exposed to a temporal afterglow which emphasizes the important role of long-live reactive species generated in the plasma [2]. In nitrogen and oxygen plasmas, metastable species present enough energy to activate and functionalize the polystyrene chains which present bond energies in the range 2.5 to $5 \mathrm{eV}$. It is well-known that plasma treatments induce four important phenomena on polymers:

- cleaning is of high importance to improve bonding. It consists in removing layers of organic contaminants present on the surface in the $[10-100 \AA]$ thickness range. For plasma treatments with an injected power of several $\mathrm{mW} / \mathrm{cm}^{2}$ the polymer has to be exposed several seconds to obtain sufficient cleaning and an increase of adhesion [3].

- etching allows the removal of a bigger quantity of matter compared to cleaning. But this ablation process may end in the degradation of the polymer.

- cross-linking of the surface is realized by the formation of free radicals by the plasma phase. It is usually obtained in inert gases. As a consequence no new functionality appears on the surface.

- functionalization consists in the formation on the surface of new chemical functions in a depth typically of several hundreds Ångströms, without affecting the bulk properties [4]. By using reactive gases in the plasma like nitrogen or oxygen, the surface becomes more polar with the production of hydroxyl, alcohol, ether, carbonyl, carboxyl, amide and amine functions.

This paper is devoted to the analysis of macroscopic and microscopic modifications obtained on atactic polystyrene thin films by using DC pulsed nitrogen or oxygen plasmas. The thin film surface is analyzed by several ways:

- macroscopic study of the wettability through the measurement of water drop contact angles by an image processing technique,

- comparison with microscopic results from X-ray photoelectron spectroscopy (XPS) and atomic force microscopy (AFM).

There are very few works using image analysis for the characterization of polymer surfaces treated by plasma. In [5] a commercial image analysis software (Semper+) is used for measuring drop areas to characterize textiles treated by plasma, whereas [6] developed a dedicated image processing algorithm based on a water drop shape model to analyse flat polymeric insulator surfaces.

In the first part of the paper, the experimental set-up is described as regards both the test conditions of polystyrene treatment and the computer vision system (from software and hardware point of view) for contact angle measure. 
In the second part, results are shown and interpreted. The final discussion opens the way towards a better understanding of the interaction mechanisms between the polymer and the DC pulsed plasma.

\section{EXPERIMENTAL SET-UP}

\section{A. Preparation of polymer thin films}

Atactic polystyrene pellets are dissolved in toluene at room temperature. Some drops of this solution are deposited on glass slides (area: $6 \mathrm{~cm}^{2}$ ) cleaned beforehand with toluene and dried with acetone. These slides are stored in a dessicator, in which the evaporation of solvent is carried out under controlled atmosphere, in order to obtain homogeneous polystyrene films with an approximate thickness of $10 \mu \mathrm{m}$.

\section{B. Technical set-up}

Fig. 1 presents the experimental set-up used for plasma treatment of atactic polystyrene, and Tab. I lists the various parameter values.

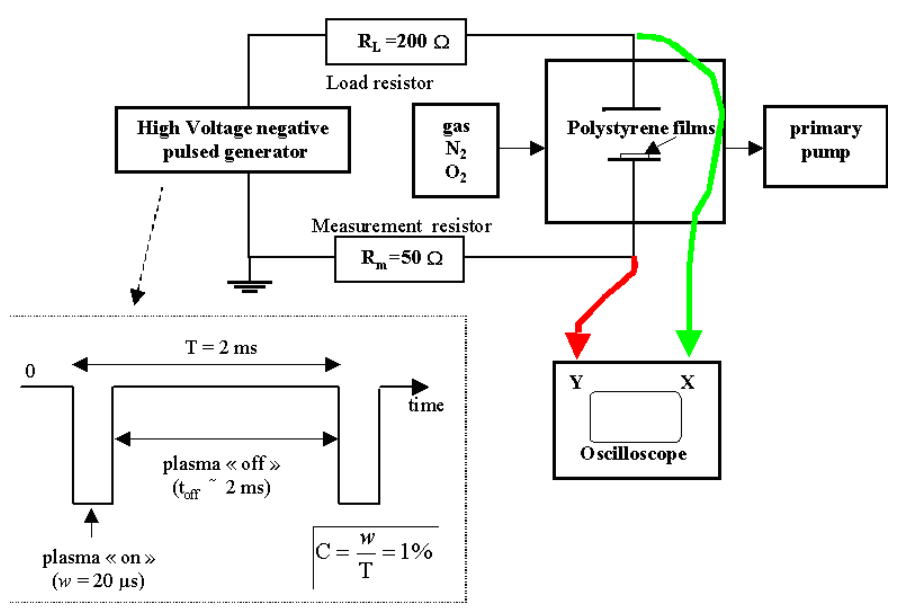

Fig. 1. Experimental set-up for plasma treatment of atactic polystyrene.

The plasma reactor consists of two stainless-steel plane electrodes (diameter: $10 \mathrm{~cm}$ ) inside a stainless-steel chamber that is pumped down to $5 \cdot 10^{-3}$ mbar before each treatment. The running pressure is $p=4$ mbar in nitrogen or oxygen (purity 99.99\%). One electrode is grounded and the other is negatively polarized using a high power DC pulsed generator. Two resistors are used in the electrical circuit: a load resistor $R_{L}=200 \Omega$ required for high voltage power supply and a measurement resistor $R_{m}=50 \Omega$. The duty cycle $C=w / T$ is set to $1 \%$, where $T$ is the pulsed voltage period, and $w$ is the pulse width i.e. the glow duration time.

\section{Electrical discharge conditions}

With these experimental conditions, a plasma corresponding to the abnormal glow discharge is generated between the two plane electrodes during each pulse. Discharge current and voltage waveforms are recorded via an oscilloscope (bandwidth of $300 \mathrm{MHz}$ and acquisition rate
TABLE I

Experimental Parameter setting

\begin{tabular}{|c|l|c|}
\hline Symbol & Meaning & Value \\
\hline \hline & Thin Film thickness & $10 \mu \mathrm{m}$ \\
\hline $\mathrm{p}$ & Runing pressure & $4 \mathrm{mbar}$ \\
\hline $\mathrm{d}$ & Gap distance between electrodes & $1 \mathrm{~cm}$ \\
\hline $\mathrm{P}$ & Fixed Average Power value & $6 \mathrm{~W}$ \\
\hline $\mathrm{t}$ & Treatment duration time & $0 \rightarrow 60 \mathrm{~s}$ \\
\hline $\mathrm{N}$ & Number of pulses & 30000 \\
\hline $\mathrm{T}$ & Period of the signal & $2 \mathrm{~ms}$ \\
\hline$\nu$ & Frequency of pulsed voltage & $500 \mathrm{~Hz}$ \\
\hline$w$ & Pulse width (Glow duration time) & $20 \mu \mathrm{s}$ \\
\hline $\mathrm{C}$ & Duty cycle & $1 \%$ \\
\hline
\end{tabular}

of $2.5 \mathrm{GS} / \mathrm{s}$ ). The total treatment duration time $t$ is controlled by a lab-made oscillator counter.

From anode to cathode, the abnormal glow discharge consists of a positive column (close to anode), a Faraday dark space and a negative glow (close to cathode). For each treatment, the polystyrene thin film is placed either on the anode or on the cathode.

The average power value is fixed at $P=6 \mathrm{~W}$, and the characteristic surface for the discharge is of about $67 \mathrm{~cm}^{2}$. Therefore the power level injected in the discharge is approximately of $90 \mathrm{~mW} / \mathrm{cm}^{2}$ which is highly sufficient to obtain cleaning of the surface and doubtless etching, crosslinking and functionalization. Since the power remains constant during treatments, results are presented as a function of the treatment duration time $t$ which is proportional to the energy injected into the plasma : $E=P \times t$.

It should be mentioned that the effective time of plasma "ON" is one hundred times smaller than the treatment duration time because of the very low duty cycle: $t=N T=$ $N\left(w+t_{o f f}\right)=t_{e g}+t_{\text {eag }}$, where $N$ is the number of pulses injected into the plasma, $w$ and $t_{\text {off }}$ are respectively the glow and afterglow duration times, $t_{e g}$ and $t_{e a g}$ are respectively the effective glow and afterglow duration times.

\section{Contact angle measurement}

The treatment is characterized by measuring the contact angle of a deionized water drop on the polystyrene surface (Fig. 2). Measurement is made immediately after treatment (delay lower than $5 \mathrm{~min}$ ). Four drops are deposited at various places on a given polymer surface with a syringe (volume of $5 \mu \mathrm{l}$ ) in order to obtain an averaged value of the contact angle. The results of wettability are given by the relative variation of the contact angle:

$$
\frac{\Delta \theta}{\theta}=\frac{\theta_{i}-\theta_{f}}{\theta_{i}}
$$

where $\theta_{i}$ and $\theta_{f}$ are respectively the initial and final contact angles (each one being an average value of four angles).

The initial contact angle is typically $\theta_{i} \cong 85^{\circ}$ (water drop almost spherical before treatment). 


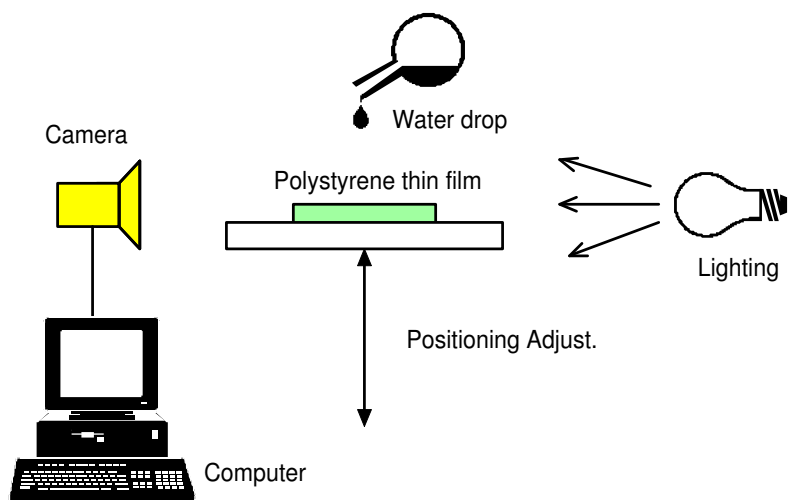

Fig. 2. Imaging system for drop contact angle measurement

\section{Image Processing System}

An image processing technique is used to measure precisely the contact angle. A grey-level image $I(x, y)$ of the drop deposited on the polymer is acquired with a video camera (ITM-M-SC Intertec components, miniature video camera: 260 lines). Fig. 3 shows the user interface with a typical drop image and the extracted contours.

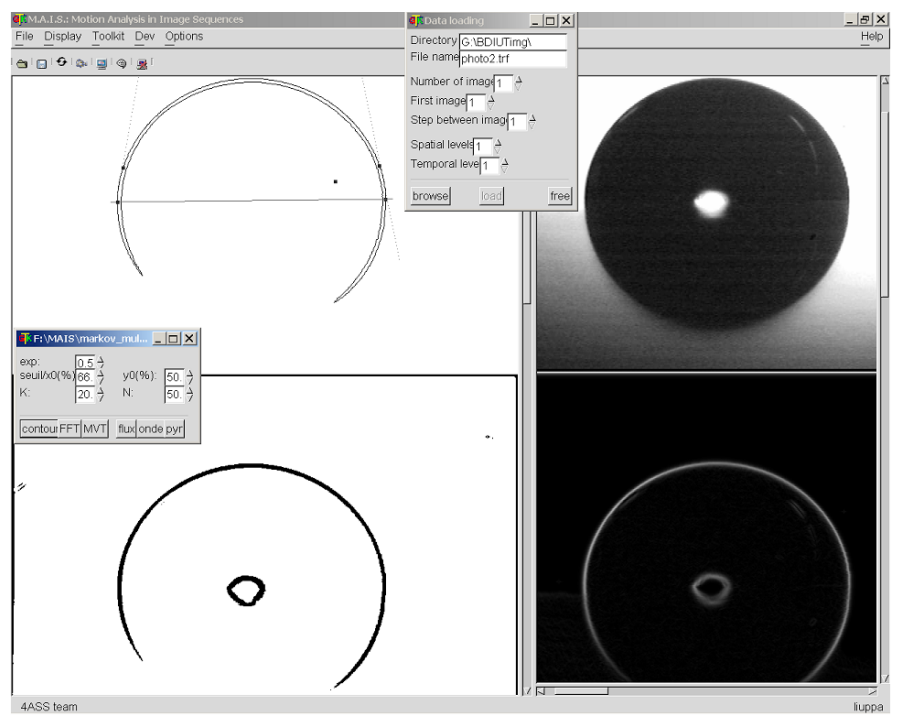

Fig. 3. Image processing system: user interface for automatic contact angle estimation (case of a non-wettable surface: the drop is spherical)

The contour detection processing consists in the following steps:

- First, the spatial (respectively vertical and horizontal) partial derivatives $\partial I / \partial x$ and $\partial I / \partial y$ are estimated using an exponential derivation filter applied respectively on lines and columns [7]. Contour detection often uses a smoothing of the image in one direction followed by a derivation in the other direction, in order to reduce noise. Here the discrete impulse response of the smoothing filter is given by:

$$
h(k)=c \cdot e^{-\alpha|k|}
$$

where $\alpha$ is a smoothing real and positive parameter (typ. $\alpha=0.5$ ) and $c=\frac{\alpha}{2}$ is a normalizing factor (for unitary step response). The impulse response of the derivation filter is the derivative of the impulse reponse of the smoothing filter, which may be written as a sum of a causal and an anti-causal term:

$$
\begin{aligned}
g(k) & =g^{+}(k)+g^{-}(k) & \text { with } \\
g^{+}(k) & =-c \alpha e^{-\alpha k} & \text { for } k>0 \\
g^{-}(k) & =c \alpha e^{\alpha k} & \text { for } k<0
\end{aligned}
$$

This leads naturally to a parallel implementation. The recursion equations for cascade implementation are given by:

$$
\begin{aligned}
y^{+}(k) & =-x(k)+e^{-\alpha}\left(y^{+}(k-1)+x(k)\right) \\
y^{-}(k) & =x(k)+e^{-\alpha}\left(y^{-}(k+1)-x(k)\right) \\
y(k) & =y^{+}(k)+y^{-}(k)
\end{aligned}
$$

They are applied on lines and columns respectively to get the two spatial derivatives.

- Secondly, the modulus of the spatial gradient $\sqrt{(\partial I / \partial x)^{2}+(\partial I / \partial y)^{2}}$ is computed (Fig. 4b) and then thresholded by using an entropic approach as described in [8]. The threshold is taken as: $\theta=\kappa \cdot 2^{H}$ where $H$ is the entropy of observations (here the gradient modulus) and $\kappa$ is a proportionality factor (typ. $\kappa=20$ for edge detection). It yields a binary map showing the detected contours (points in black on Fig. 4c).

- Then the binary contour tracker of Rosenfeld and Kak [9] is run to extract the set of connected pixels forming the exact frontier of the drop: we get a vector containing the indices of the contour points (chain-list), with their $x$ - and $y$-coordinates. Note that for initialization of the contour tracker, the position of a starting point should be taken near or inside the drop area (automatic or manual positioning).

- From this list, the triple points corresponding to $x_{\max }$ and $x_{m i n}$ are easily extracted and the tangents to the drop at those two points are estimated by a least-squares algorithm taking into account $N$ neighboring points (typ. $N=50)$ such that $y<y\left(x_{\max }\right)$ and $y<y\left(x_{\min }\right)$. Indeed, the origin of the image is classically taken in the upper-left corner. The base-line joining the two triple points is also automatically extracted and plotted (Fig. 4d).

- Finally, the angle is measured as the average value of the two angles on both sides of the drop (Fig. 4e).

This processing is iterated on a batch of drop images, without any further interaction with the end-user: default parameter values are set once and for all at the beginning of the process.

Worth noticing is the fact that the measurement process is entirely automatic and the precision achieved by this image processing technique is better than $1.5^{\circ}$ (far below the total measurement error which is of about $5^{\circ}$, due to the acquisition system: bad manual focusing). Moreover, this reliable method for measuring contact angles is very low cost. For comparison purpose, the total measurement error was almost of $10^{\circ}$ with former manual estimation (projection of the image on a white screen and use of a protractor) and it was highly dependent on the user ability. 


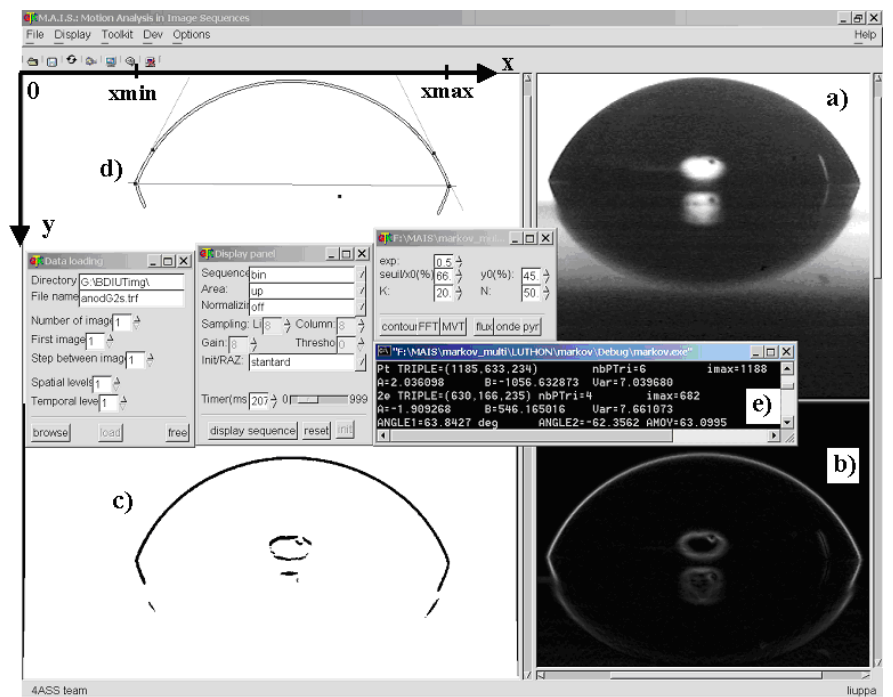

Fig. 4. Contact angle measurement by image processing (case of a wettable surface: the drop is flattened): a) original image of the drop deposited on the polymer; b) modulus of the spatial gradient; c) binary map of detected contours; d) plot of the two tangents and the base-line; e) automatic computation of the contact angle

The image processing software was developed in $\mathrm{C} / \mathrm{C}++$ with the GTK graphical library. The application is portable both under Windows and Linux system. A setup program is furnished to the client user for installation of the stand-alone executable version on a PC.

\section{Experimental Results}

\section{A. Macroscopic analysis: wettability changes}

The effect of plasma treatment duration time $t$ has been studied in nitrogen when thin films are deposited close to the negative glow or the positive column. Results are presented in Fig. 5a where a well-known behaviour is observed: the wettability of the surface increases as a function of $t$ to reach a plateau after $\approx 20$ seconds, where the contact angle relative variation $\Delta \theta / \theta$ gets close to 0.7 .

Moreover a shoulder appears on the curves, that depends on the position of the polymer in the glow discharge. When the polymer is placed close to the positive column the amplitude of the shoulder is weak and it is obtained for $t=6 \mathrm{~s}$. When the polymer is placed close to the negative glow the shoulder is obtained for $t=10 \mathrm{~s}$. This shoulder on the contact angle curve has already been observed in plane to plane and point to plane electrode configurations. Particularly remarkable, this phenomenon indicates a partial hydrophobic recovery of the surface during plasma processing.

This shoulder phenomenon also depends on the nature of the gas used in the plasma (nitrogen or oxygen). Fig. 5b shows the contact angle relative variation in oxygen. At $t=6 \mathrm{~s}$, weak shoulders can be observed on the curves whatever the polymer position is. The amplitude of these shoulders is very low compared to the treatments performed in nitrogen.

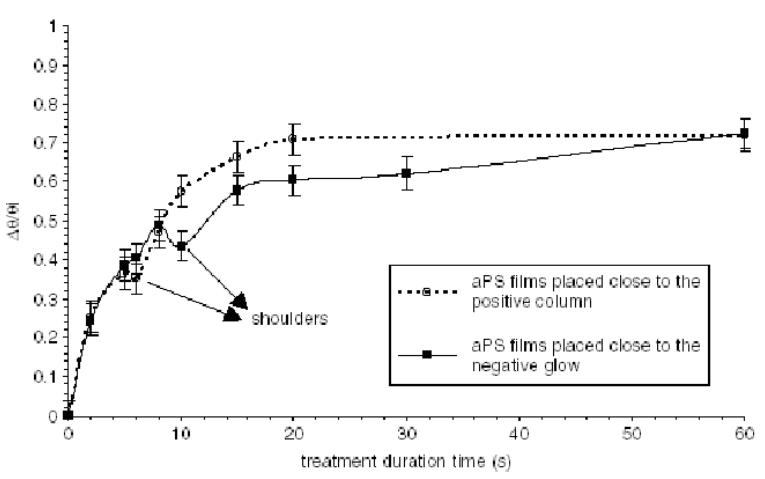

a)

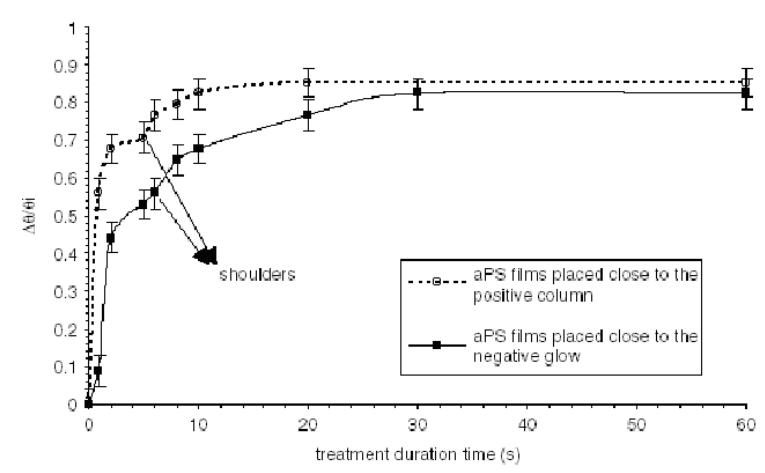

b)

Fig. 5. Wettability curves: Contact angle relative variation $\Delta \theta / \theta$ as a function of the treatment duration time $t$, for the two positions of polystyrene films and in the two gases: a) in nitrogen b) in oxygen.

To summarize the conditions for which a wettability shoulder is observed, it can be stated that the amplitude of this phenomenon is mainly depending on the gas nature (higher in nitrogen than in oxygen) but also on the position (higher when the polymer is deposited close to the negative glow rather than to the positive column).

Moreover the temporal position of this shoulder seems to be earlier $(\approx 6 \mathrm{~s})$ both in oxygen and nitrogen plasma when the polymer is placed close to the positive column of the glow discharge. It seems to take place latter $(\approx 10 \mathrm{~s})$ in nitrogen plasma when the polymer is placed close to the negative glow.

Nevertheless and whatever the gas used, this shoulder appears for a treatment duration time in the range 6 to $10 \mathrm{~s}$. This treatment duration time seems to be characteristic of the surface state: we will denote it $t_{c}$ in the following.

\section{B. Comparison with Microscopic analyses}

\section{B.1 X-ray Photoelectron Spectroscopy: Chemical Changes}

XPS spectra were recorded using a spectrometer delivering a monochromatic X-ray $(1486.6 \mathrm{eV})$ used for excitation. The binding energies were determined by quantitative analyses, using the $C 1 s$ binding energy of contamination carbon $(284.6 \mathrm{eV})$ as the reference, with an experimental error of $\pm 0.2 \mathrm{eV}$.

Before treatment, polystyrene films were analyzed to 
know the initial chemical structure of the surface. The polymer surface is only composed of carbon (Fig. 6): the

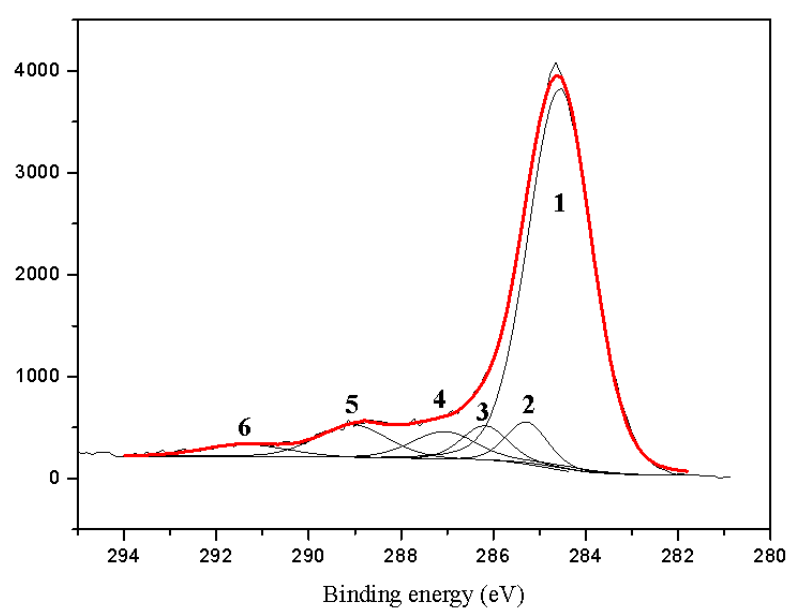

Fig. 6. Example of $C 1 s$ core level spectrum obtained for atactic polystyrene thin films treated with $N_{2}$ plasma.

main peak (1) at $284.6 \mathrm{eV}$ corresponds to the aliphatic and aromatic carbon and the shake-up peak (6) at $291.2 \mathrm{eV}$ is characteristic of the aromaticity of the polystyrene. After treatment with nitrogen or oxygen plasma, new peaks of weak intensities are observed that correspond to new surface functionalities due to plasma exposure: amine $\mathrm{C}-\mathrm{N}$ (2), C-O (3), carbonyl $\mathrm{C}=\mathrm{O}(4)$ and carboxyl O-C=O (5).

Fig. 7 shows quantitative results. The nitrogen plasma introduces on the surface more oxygen bonds than nitrogen bonds: the $\mathrm{N} / \mathrm{C}$ atomic ratio increases very slowly towards a weak value of 0.05 , whereas the evolution of the $\mathrm{O} / \mathrm{C}$ atomic ratio is noticeable. This well-known result [4] may be attributed to residual oxygen and water vapour contained in the reactor during the treatment or to reactions with oxygen during the sample exposure to atmosphere. When the polymer is deposited close to the negative glow, the $\mathrm{O} / \mathrm{C}$ ratio increases in the $[0.1-0.15]$ range during the first $10 \mathrm{sec}$ of treatment to reach a final value of 0.2 . When it is deposited close to the positive column the $\mathrm{O} / \mathrm{C}$ ratio is close to 0.2 over the whole treatment duration time range.

As regards results with oxygen plasma, O/C atomic ratio curves evolve similarly as with nitrogen, but towards a higher limit of about 0.3 .

Finally, whatever the gas used and the position of the polymer, it is observed that in the wettability shoulder time interval [6-10s], numerous weak shoulders appear in the $\mathrm{O} / \mathrm{C}$ and $\mathrm{N} / \mathrm{C}$ composition ratios.

\section{B.2 Atomic Force Microscopy: Morphological Changes}

We imaged polystyrene samples in ambient conditions using a commercial AFM head. The surface is characterized by the measure of its root-mean squared roughness $R m s$, that is estimated over the whole image sample (dimension $5 \times 5 \mu \mathrm{m})$.

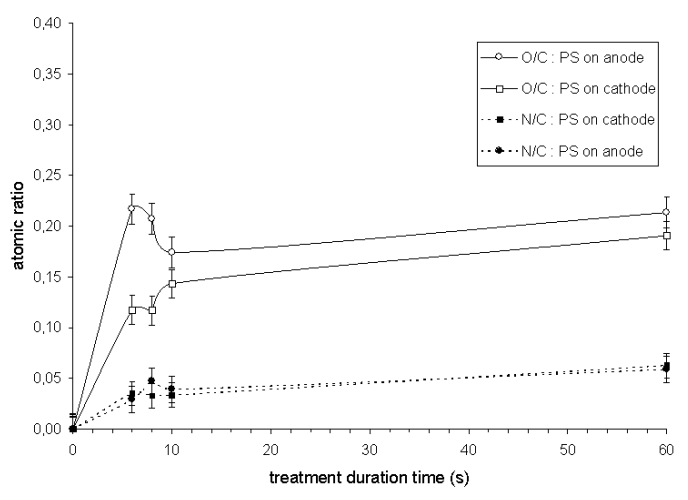

Fig. 7. Atomic ratios $\mathrm{O} / \mathrm{C}$ and $\mathrm{N} / \mathrm{C}$ as a function of the treatment duration time $t$ when the polystyrene thin films are deposited close to the negative glow (cathode) or close to the positive column (anode) in a $N_{2}$ plasma

This measurement gives the signature of morphological modifications of the surface occurring with plasma treatment. Images were recorded in different zones in order to be representative of the total sample surface state.

Fig. 8 shows the Rms variations and the atomic ratio $\mathrm{O} / \mathrm{C}$ of oxygen grafted on the surface as a function of $t$ (treatment in nitrogen, thin film on cathode).

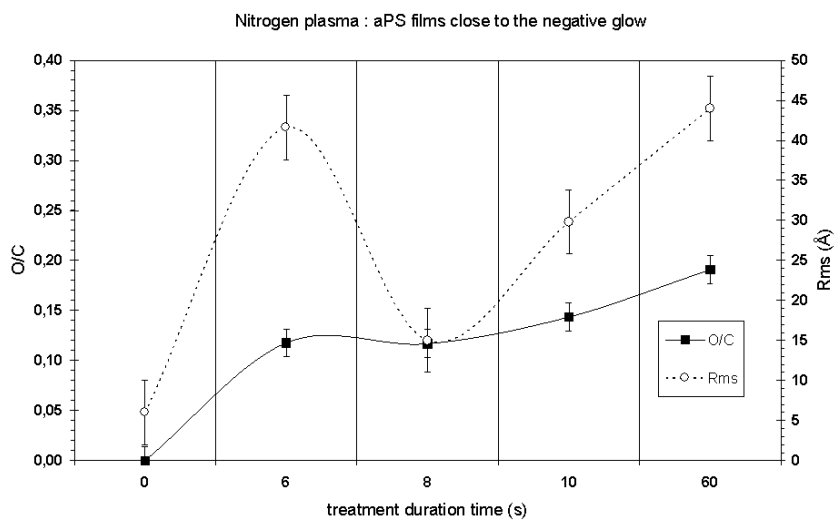

Fig. 8. Roughness $R m s$ and atomic ratio $\mathrm{O} / \mathrm{C}$ as a function of the treatment duration time $t$ in a nitrogen plasma, when the polystyrene films are deposited on the cathode

The untreated polymer in initial state presents a very smooth surface with $R m s=6 \AA$.

It can be observed that in the wettability shoulder time interval [6s-10s], a decrease of Rms corresponding to a smoother surface occurs for $t_{0}=8 \mathrm{~s}$. Afterwards, the roughness value increases slowly but never exceeds $R m s_{\max }=50 \AA$ which still corresponds to a smooth surface after plasma treatment. The morphological modifications due to the plasma are thus limited to the extreme surface in several $10 \AA$ in depth. Consequently we make the following statement: the plasma mean effect on the polymer is chemical and not physical. The important improvement of wettability may thus be explained by surface grafting of numerous oxygenated polar functions even if the 
gas used for the treatment is nitrogen.

Moreover the polymer surface smoothness at $t_{0}$ is more important for treatment in nitrogen plasma close to the negative glow which may be correlated to the higher wettability shoulder in the same conditions. At $t_{0}=8 s$ one obtains an atomic ratio $\mathrm{O} / \mathrm{C}$ of nearly 0.1 . According to these results it can be suggested that the weak loss of roughness within the wettability shoulder time interval obtained at $t_{0}$ is due to the removing of matter on the extreme surface.

\section{Correlation Between macroscopic AND MICROSCOPIC ANALYSES: INTERPRETATION}

The polystyrene surface main modification due to the plasma is macroscopic and corresponds to a significant improvement of wettability. In the same time microscopic surface changes are weak and occur on the first macromolecular layers of the polymer (typical depth of several tens to several hundreds of Ångströms, $c f$. AFM and XPS analyses [4]).

Recalling the four important phenomena that a plasma realizes on polymer (section I), a macroscopic explanation may be given concerning the interaction between the polystyrene surface and the DC pulsed plasma. First, it can be suggested that in our experimental conditions, the surface etching is negligible since the roughness measures stay always below $50 \AA$, which corresponds to a smooth surface. Secondly, since the gases used for treatment (nitrogen or oxygen) are not inert gases like argon or helium, the cross-linking (by activated species of inert gases) may also be neglected.

Thirdly, according to the curve of wettability (Fig. 5) the shoulder appears for the characteristic treatment duration time $t_{c} \approx 8 s$. In the $\left[0, t_{c}\right]$ time interval, the plasma creates numerous reactive species which collide with the surface and have enough energy to break and react with the covalent bonds of the polystyrene. They weaken the surface and realize rapidly the surface cleaning. Recall here that the effective glow discharge time is equal to: $t_{e q}=C \times t_{c}$. Thus during this first rapid treatment stage, the cleaning consists in removing layers of organic contaminants which are in the same time oxidized. The hypothesis of a cleaning stage in the treatment is coherent with the literature [3]. The $[10-100 \AA]$ thickness range of known cleaning effect is in agreement with our results: the $R m s$ never exceeds 50 $\AA$. Moreover the power level of $90 \mathrm{~mW} / \mathrm{cm}^{2}$ injected in the discharge is widely sufficient to remove the organic contaminants: usually for plasma treatments with power injected of several $\mathrm{mW} / \mathrm{cm}^{2}$ the polymer has to be exposed many seconds to obtain sufficient cleaning. With our treatment, the power level is nearly a hundred times higher than in the literature but the effective treatment duration times are nearly a hundred times lower (duty cycle of $1 \%$ ).

At treatment time $t_{c}$ it can be supposed that the surface is in a new initial state and the treatment corresponding mainly to the surface functionalization begins: the time interval $\left[t_{c}, 60 \mathrm{~s}\right]$ concerns the improvement of the macroscopic surface properties and is the second stage of treatment. In the same time, we could suppose a slow etching of the surface because of the weak increase of roughness.

To sum up, this characteristic treatment time $t_{c}$ seems to be the necessary time to remove all the organic and oxidized contaminants of the surface. This time depends on the plasma conditions which correspond to the energy levels injected in the gas.

\section{Conclusion}

A macroscopic interpretation of the polystyrene treatment by a DC pulsed plasma has been described in this paper. The treatment consists in two main stages: the cleaning of the surface and its functionalization. The macroscopic characterization of the polystyrene surface (wettability curves) was carried out thanks to an automatic imaging system that yields robust and fast measurements of contact angles of water drops deposited on the polymer.

Using a DC pulsed plasma with very low duty cycle for polymer surface treatment presents a big interest. Economically the effective glow discharge duration time is one hundred times lower than with usual techniques. Moreover the industrial applications like the improvement of the adhesion may be obtained in soft conditions without degradation of the polymer. Note that nowadays, the same kind of treatment may be carried out for industrial purpose at atmospheric pressure, which eliminates a strong drawback and opens the field to a wide range of new low-cost applications, either in medicine (for contact lenses, pace-makers, sterilization), in aeronautics (for materials like titane), or in wood and cotton industry (treatment against mouldiness, hydrophobicity).

\section{ACKNOWLEDGMENT}

The authors thank C. Guimon, H. Martinez and Y. Tison from University of Pau, France, for XPS and AFM results.

\section{REFERENCES}

[1] M.R. Wertheimer, "Plasma treatment of polymers and other materials for improved adhesion," in EURADH Congress, Freiburg im Breisgau, Germany, Sept. 2004, pp. 85-91.

[2] J. Larrieu, B. Held, H. Martinez, and Y. Tison, "Ageing of atactic and isotactic polystyrene thin films treated by oxygen DC pulsed plasma," Surface and Coatings Technol., vol. 200, pp. 2310-2316, 2005.

[3] E.M. Liston, L. Martinu, and M.R. Wertheimer, "Plasma surface modification of polymers for improved adhesion: a critical review," J. Adhesion Sci. Technol., vol. 7, no. 10, pp. 1091-1127, 1993.

[4] C.M. Chan, T.M. Ko, and H. Hiraoka, "Polymer surface modification by plasmas and photons," Surface Science Reports, vol. 24, no. 1-2, pp. 1-54, 1996.

[5] G. Allan, A. Fortheringham, and P. Weedall, "The use of plasma and neural modelling to optimise the application of a repellent coating to disposable surgical garments," AUTEX Research Journal, vol. 2, no. 2, pp. 64-68, June 2002.

[6] M. Berg, R. Thottappillil, and V. Scuka, "Hydrophobicity estimation of $\mathrm{HV}$ polymeric insulating materials: Development of a digital image processing method," IEEE Trans. Dielectrics and Electrical Insulation, vol. 8, no. 6, pp. 1098-1107, Dec. 2001.

[7] S. Castan, J. Zhao, and J. Shen, "New edge detection methods based on exponential filter," in 10th Int. Conf. on Pattern Recognition, Atlantic City, NJ USA, June 1990, pp. 709-711, Vol.1.

[8] F. Luthon, M. Liévin, and F. Faux, "On the use of entropy power for threshold selection," Signal Processing, vol. 84, pp. 1789-1804, 2004.

[9] A. Rosenfeld and A. Kak, Digital Picture Processing, Academic Press, 1976. 